\title{
Defect Reports Classification with pre-trained BERT
}

\author{
Rin Hirakawa ${ }^{a^{*}}$, Keitaro Tominaga ${ }^{\mathrm{b}}$, Yoshihisa Nakatoh ${ }^{\mathrm{a}}$ \\ ${ }^{a}$ Kyushu Institute of Technology, 1-1, Sensui-cho, Tobata-ku, Kitakyushu-shi, Fukuoka, 804-0015, Japan \\ bPanasonic System Design Co., Ltd., 3-1-9, Shinyokohama, Kohoku-ku, Yokohama City, 222-0033, Japan \\ *Corresponding Author: nakatoh@ecs.kyutech.ac.jp
}

\begin{abstract}
In recent years, the movement to use OSS to various functions has become active. While various benefits can be obtained by using OSS, there is also a disadvantage that it is difficult to prevent problems caused by OSS in advance. In order to analyze defects, it is important to classify defect reports into appropriate categories quickly and accurately. However, with the rapid expansion of the development scale of OSS in recent years, the number of defects has become enormous, and the time taken to classify defects has increased. In this paper, in order to solve such problems, we propose a system that automatically classifies defect reports into appropriate categories using the neural language model BERT. In classification experiments using actual defect reports, we verify the effectiveness of proposed method. The $\mathrm{F}$ value was 0.86 , and the reports could be classified with high accuracy.
\end{abstract}

Keywords: OSS, Defect Report, Classification, NLP, BERT.

\section{Introduction}

Recently, OSS (Open Source Software) is utilized in various fields. Using them, various merits can be obtained such as shortened development cycle, acquisition of new functions by updating, and improvement of internal implementation transparency. On the other hand, there is also a disadvantage that defects due to the following two factors are likely to occur.

- Revelation of problems that exist inside OSS

- Improper use and modification of OSS

Such problems are difficult to prevent in advance. Therefore, in the system development using OSS, the person in charge of the defect improvement classifies the "defects ticket" issued from the defect management system into the appropriate category to facilitate the defect analysis.
Although the speed and accuracy of defect classification are required for early solution of defects, the number of defects becomes huge as the development scale of OSS increases, so the time to perform classification puts a burden on the project. We try to improve speed and accuracy by automating defect classification, in order to reduce the time for defect classification commonly held by system development utilizing OSS. In this paper, we propose a system that uses pre-trained neural language model to automatically classify defect reports into categories. In the experiment, we verify the effectiveness of the system using actual defect reports data.

\section{Proposed Method}

\subsection{Overview}

Our proposed system takes as input a "ticket" created on a defect management system such as Redmine. The tester of the system describes the defect that occurred at the time of the test in the defect report with the comment and uploads it on the defect management system. Reports aggregated into a defect management system do not have information as to which OSS built into the system is the cause of the defect. In conventional system development, categorization was performed manually based on the content of the defect report, and the category information was added to the ticket. However, such a method requires a large number of personnel in case of large-scale system development, and there is a problem that the time cost becomes enormous. In addition, in a large-scale system using OSS, knowledge gained through many years of experience is required to determine the root cause of errors. Therefore, it is difficult to gather personnel that can be classified with the same degree of accuracy. This part in our system is replaced with a classifier based on the neural language model BERT ${ }^{(1)}$ to automate and speed up development process (Fig 1). 


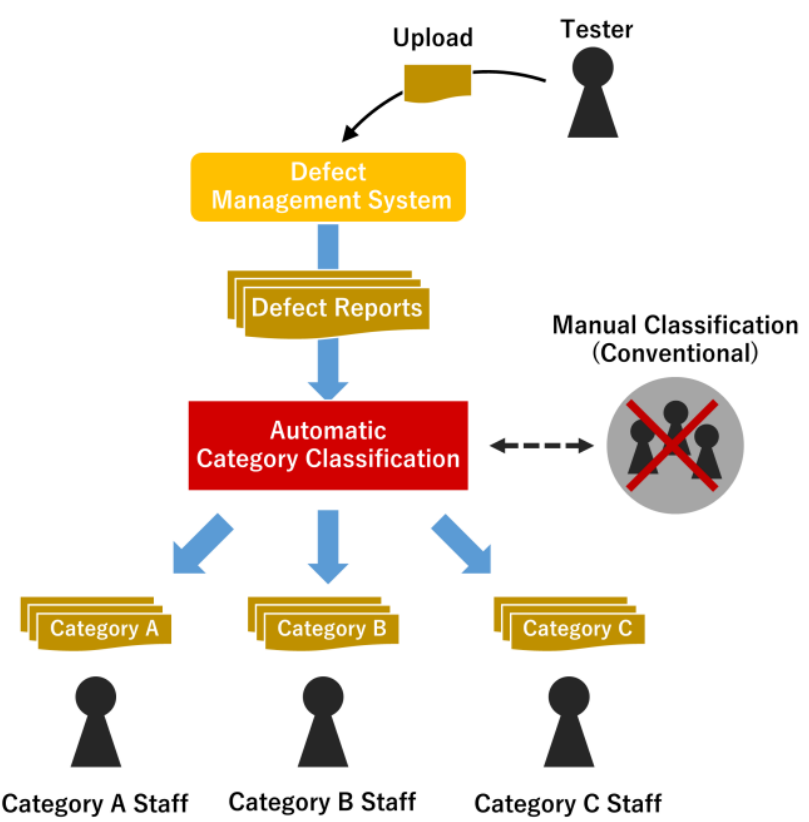

Fig. 1. Flow of Proposed System.

\subsection{BERT}

BERT $^{(1)}$ (Devlin et al., 2018) is the general purpose neural language model that can be applied to various natural language tasks after fine-tuning. Devlin et al made it possible to train bi-directional Transformer by using a method called Masked LM, and achieved SOTA in eight NLP tasks. Google has published BERT models pre-trained with Wikipedia and Books Corpus, and researchers around the world are trying to apply BERT to their language tasks. Since BERT consists of multi-head attention, it has the advantage of being able to visualize the part that the model is watching in predictions. This feature is very useful as we need to know what information is considered in the classification of defect reports.

\subsection{WordPiece Tokenizer}

WordPiece $^{(2)}$ is a method that can solve the problem of unknown words by decomposing low frequency vocabulary into characters or partial strings. The WordPiece model is trained by unsupervised learning based on $\mathrm{BPE}^{(3)}$ (Byte Pair Encoding) which minimizes the number of divisions for the corpus. The advantage of using WordPiece is that it can handle words not in vocabulary, spelling variations, typographical errors as meaningful information. The actual defect reports contain a large number of words expressed in camel-case and programming language-specific vocabulary, and by treating them as sub-words, the defect description can be more flexibly interpreted.

\section{Experiment}

In order to confirm the effectiveness of the proposed method, we will conduct categories classification experiments using the data of actual defect reports. We will first describe the dataset used in the experiment, and then explain the experimental method and conditions.

\subsection{Dataset}

We use a part of Rediscovery Datasets ${ }^{(4)}$ for classification experiments. The datasets contain defect report data of three OSS projects (Apache, Eclipse, and KDE), which are mined on Bugzilla, one of the defect tracking systems. These data were collected from 1999 to 2017 and consist of about 914 thousand defect reports. We conduct experiment using Eclipse defect report data.

\subsection{Preprocessing}

Defect reports contain information such as product, reporter, version, and defect description. In this experiment, only the defect description is used to predict the class of the defective product. Prior to experimentation, data that contains NA values or duplicates are removed. Also, data that the number of its character is less than 10 are deleted because it is too short to be interpreted and may adversely affect learning of product classes. Table 1 shows the details of the statistical information of the defect description. Also, classes are sorted by the number of included data, leaving only the defect reports that belong to the top five classes. The details of the data are shown in Table 2.

\subsection{Method}

In this experiment, a part of defect report data is used to fine-tune the BERT model, and the remaining data are used to verify classification accuracy. The following describes the experimental procedure.

Table 1. Statistical information of the defect description.

\begin{tabular}{|c|c|}
\hline Statistics & \# of characters \\
\hline Mean & 57 \\
\hline Std & 22 \\
\hline Min & 1 \\
\hline 25 percentile & 42 \\
\hline 50 percentile & 55 \\
\hline 75 percentile & 70 \\
\hline Max & 255 \\
\hline
\end{tabular}


Table 2. Contents of Eclipse Defect Report.

\begin{tabular}{|c|c|c|}
\hline Label & $\begin{array}{c}\text { Product Class } \\
\text { Name }\end{array}$ & \# of data \\
\hline$(0)$ & Platform & 109096 \\
\hline$(1)$ & JDT & 54771 \\
\hline$(2)$ & z_Archiv & 29737 \\
\hline$(3)$ & BIRT & 22655 \\
\hline$(4)$ & CDT & 19676 \\
\hline
\end{tabular}

(a) Split the Data

The entire data are divided into 6:2: 2 and use each for training, validation, and testing.

(b) Fine-tune BERT model

Fine-tune the BERT model using the training data above. The conditions for fine-tuning and the parameters of the model are shown in Table 3.

(c) Classify Test Data

Test data are classified by BERT model after fine-tuning and calculate score.

Table 3. Finu-tuning Parameters.

\begin{tabular}{|c|c|}
\hline Parameter & Value \\
\hline Pre-trained Model & bert-base-uncased \\
\hline Max Sequence Length & 128 \\
\hline Train Batch Size & 6 \\
\hline Learning Rate & $5 \mathrm{e}-6$ \\
\hline Epochs & 10 \\
\hline $\begin{array}{c}\text { Cyclical Learning Rate } \\
\text { Type }\end{array}$ & $\begin{array}{c}\text { warmup cosine } \\
\text { hard restarts }\end{array}$ \\
\hline Vocab Size & 30522 \\
\hline
\end{tabular}

\section{Result}

Fig. 2 shows a confusion matrix obtained from the classification result of test data, the vertical axis represents true labels, and the horizontal axis represents predicted labels. Table 4 shows the number of data for each class and the classification scores calculated based on the confusion matrix. According to the confusion matrix, it can be seen that the data in each class is likely to be misclassified into the JDT and Platform. These are top of two classes which have the largest number of data.

In addition, the CPU time required for classification for all 47071 test data was 696.5 seconds, and it took an average of 0.015 seconds per case. The classification experiment was performed on a PC equipped with one graphics card NVIDIA GeForce GTX TITAN Black. This result means that the time to classify defect report data has been reduced from several days to a few minutes. By using this system, developers will be able to devote a lot of time to fixing bugs instead of defect reports classification.

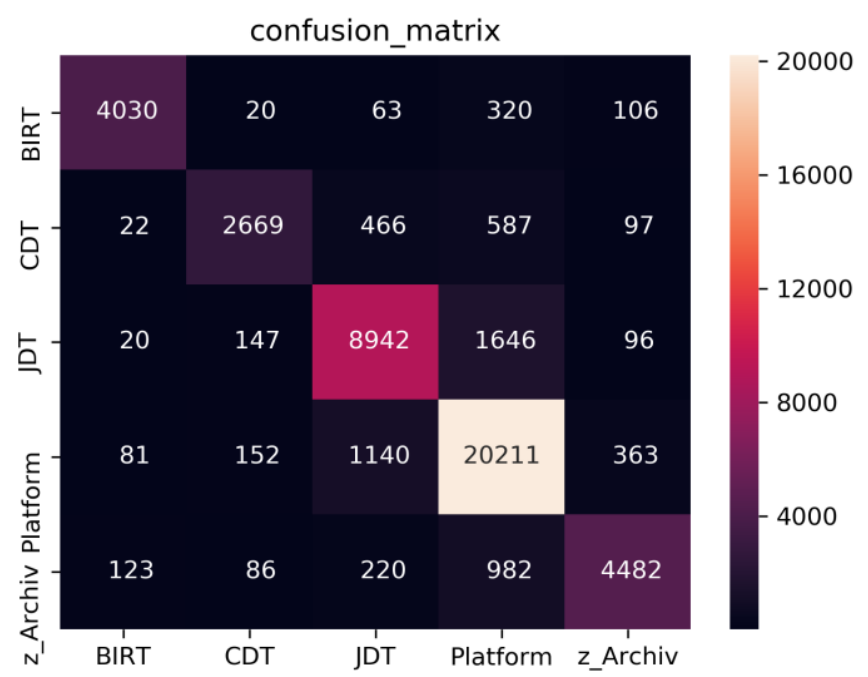

Fig. 2. Confusion Matrix of Category Classification.

Table 4. Classification Scores.

\begin{tabular}{|c|c|c|c|c|c|}
\hline & & Precision & Recall & F1-Score & Support \\
\hline \multirow{5}{*}{ Product } & BIRT & 0.94 & 0.89 & 0.91 & 4539 \\
\hline & CDT & 0.87 & 0.69 & 0.77 & 3841 \\
\hline & JDT & 0.83 & 0.82 & 0.82 & 10851 \\
\hline & Platform & 0.85 & 0.92 & 0.88 & 21947 \\
\hline & z_Archiv & 0.87 & 0.76 & 0.81 & 5893 \\
\hline \multicolumn{2}{|c|}{ Micro Average } & 0.87 & 0.86 & 0.86 & \multirow{3}{*}{47071} \\
\hline \multicolumn{2}{|c|}{ Weighted Average } & 0.86 & 0.86 & 0.86 & \\
\hline \multicolumn{2}{|c|}{ Accuracy } & \multicolumn{3}{|c|}{0.86} & \\
\hline
\end{tabular}




\section{Discussion}

Table 5 shows 10 defect report descriptions randomly extracted from misclassified test data. The Truth and Pred numbers correspond to the label numbers in Table 2. Some tokenized descriptions show that domain specific vocabularies like "TestNG" can be treated well as subwords.

Table 5. Randomly Sampled Misclassified Data.

\begin{tabular}{|c|c|c|}
\hline \multicolumn{2}{|c|}{ Label } & \multirow{2}{*}{ Tokenized Discription } \\
\hline Truth & Pred & \\
\hline (1) & (2) & $\begin{array}{l}\text { [CLS] format action does not } \\
\text { appear for } 1 \text { st level items in } \\
\text { expressions view [SEP] [PAD] } \\
{[\mathrm{PAD}] . .}\end{array}$ \\
\hline (3) & (2) & $\begin{array}{l}\text { [CLS] test \#\#ng eclipse issue after } \\
\text { clicking on run \#\#as - - > test \#\#ng } \\
\text { test - an exception stack trace is not } \\
\text { available. [SEP] [PAD] [PAD] ... }\end{array}$ \\
\hline (4) & (3) & $\begin{array}{l}\text { [CLS }] \text { code does not handle errors } \\
\text { in extension definitions }[\mathrm{SEP}] \\
{[\mathrm{PAD}][\mathrm{PAD}] \ldots}\end{array}$ \\
\hline (1) & (2) & $\begin{array}{l}{[\text { CLS }] \text { exception in log when }} \\
\text { working with java projects }[\mathrm{SEP}] \\
{[\mathrm{PAD}][\mathrm{PAD}] . .}\end{array}$ \\
\hline (4) & (3) & $\begin{array}{l}\text { [CLS }] \text { problems with editing info } \\
\text { on a plug - in [SEP] }[\mathrm{PAD}] \\
{[\mathrm{PAD}] \ldots}\end{array}$ \\
\hline (3) & (4) & $\begin{array}{l}{[\mathrm{CLS}] \text { ref \#\#act \#\#orin \#\#g assets }} \\
\text { packages del \#\#ete \#\#s images } \\
{[\mathrm{SEP}][\mathrm{PAD}][\mathrm{PAD}] . . .}\end{array}$ \\
\hline$(0)$ & (3) & $\begin{array}{l}\text { [CLS] game gets stuck on entering } \\
\text { the play now option in main menu } \\
{[\mathrm{SEP}][\mathrm{PAD}][\mathrm{PAD}] \ldots}\end{array}$ \\
\hline (4) & (3) & $\begin{array}{l}\text { [CLS] getting unix \#\#pro \#\#ces } \\
\# \# \text { s . fork \#\#and \#\# ex \#\# ec native } \\
\text { error : argument list too long [SEP] } \\
{[\mathrm{PAD}][\mathrm{PAD}] \text {... }}\end{array}$ \\
\hline (2) & (3) & $\begin{array}{l}\text { [CLS] warnings in } \mathrm{n} \# \# 200 \# \# 6 \\
\# \# 10 \# 28-001 \# \# 0[\mathrm{SEP}][\mathrm{PAD}] \\
{[\mathrm{PAD}] \ldots}\end{array}$ \\
\hline (4) & (3) & $\begin{array}{l}\text { [CLS] long running script makes } \\
\text { output \#\#view un \#\#res \#\#pon } \\
\# \# \text { sive [SEP] [PAD] [PAD] ... }\end{array}$ \\
\hline
\end{tabular}

On the other hand, in misclassified descriptions, there is a tendency that commonly used vocabulary such as "unresponsive" is decomposed into substrings that do not make sense. The influence of over-divided vocabulary on classification needs to be investigated further in the future.

\section{Conclusions}

In this paper, we proposed a method to classify defect reports using the fine-tuned BERT model, and verified the effectiveness of the proposed method using actual defect report data. The experimental results show that the $F$ value is 0.86 , and that the defect report can be classified with high accuracy.

As a future task, we plan to develop a method to present the part of description important on the classification using attention weight that the BERT model outputs at the time of prediction. In addition, it is necessary to investigate the effect of preprocessing on classification when inputting a defect report into fine-tuned BERT model.

\section{Acknowledgment}

We thank Keiichi Tokuyama and his section members for their helpful feedback on the paper. This work is supported by a grant from Panasonic System Design.

\section{References}

(1) Jacob Devlin, Ming-Wei Chang, Kenton Lee, and Kristina Toutanova : "BERT: Pre-training of deep bidirectional transformers for language understanding”, arXiv preprint, arXiv:1810.04805, 2018

(2) Yonghui Wu, Mike Schuster, Zhifeng Chen, Quoc V. Le, Mohammad Norouzi, Wolfgang Macherey, Maxim Krikun, ... , Jeffrey Dean : “Google's Neural Machine Translation System: Bridging the Gap between Human and Machine Translation", arXiv preprint, arXiv:1609.08144v2, 2016

(3) Rico Senrich, Barry Haddow and Alexandra Birch : "Neural Machine Translation of Rare Words with Subword Units", arXiv preprint, arXiv:1508.07909v5, 2016

(4) Sadat, Mefta, Bener, Ayse Basar, \& Miranskyy, Andriy V. (2017). Rediscovery Datasets: Connecting Duplicate Reports of Apache, Eclipse, and KDE [Data set]. Zenodo. http://doi.org/10.5281/zenodo.400614 